\title{
Análisis de la resiliencia en personas divorciadas, según su nivel educativo e ingresos económicos*
}

\author{
Analysis of resilience in divorced people according \\ to their educational and income levels
}

Recibido: 8 de enero de 2016/Aceptado: 12 de octubre de 2016

http://doi.org/10.17081/psico.20.37.2425

\author{
Victoria Eugenia Cabrera-García ${ }^{1}$, Karla Tatiana Casas Rojas ${ }^{2}$ \\ Sandra Liliana Pardo Hernández ${ }^{3}$, Diana Margareth Rodríguez Martíne $z^{4}$ \\ Universidad de la Sabana, Colombia
}

Palabras clave:

Resiliencia, Divorcio, Nivel educativo, Ingresos económicos.

Key words:

Resilience, Divorce, Education level, Economic income.

\begin{abstract}
Resumen
El propósito de esta investigación fue identificar los niveles de resiliencia en personas divorciadas de acuerdo con el nivel educativo y los ingresos económicos. Para Gómez (2010), las situaciones adversas se afrontan mejor con adecuados niveles de resiliencia. El estudio contó con 139 participantes divorciados, hombres (44,6 \%), y mujeres (55,4\%); con estudios básicos (48,2\%), con estudios universitarios $(51,8 \%)$, con ingresos económicos inferiores a $\$ 1.800 .000(60,4 \%)$, y superiores a $\$ 1.800 .000$ (39,6 \%). Se utilizó una metodología cuantitativa, con diseño transversal, se corrieron Análisis de Varianza (ANOVA) con el fin de responder a las preguntas de investigación. Las personas con educación superior mostraron mayores niveles de resiliencia en competencia social y apoyo social, así mismo, los que tenían mayores ingresos económicos mostraron mayor fortaleza y confianza en sí mismos, competencia social, apoyo familiar y estructura; a su vez, se encontró un efecto de interacción entre el nivel de ingresos económicos y el nivel educativo en fortaleza y confianza en sí mismos. Para futuras investigaciones se sugiere profundizar en estos resultados a nivel cualitativo.
\end{abstract}

\begin{abstract}
The purpose of this research was to identify levels of resilience in divorced people according to their education and income levels. For Gomez (2010), adverse situations are best dealt with adequate levels of resilience. The study involved 139 divorced participants: divorced men $(44.6 \%)$ and women $(55.4 \%)$; with basic education $(48.2 \%)$, college educated $(51.8 \%)$, with an income of less than COP $\$ 1.8$ million (60.4\%) and with an income higher than COP \$1.8 million (39.6\%). The methodology was quantitative with a cross design and ANOVAS were run in order to answer the research questions. People with college education showed higher levels of resilience in social competence and social support. Furthermore, those with higher income showed greater strength and self-confidence, social competence, family support and structure. In addition, an effect was found between the interaction of levels of income and education level, and the in strength and self-confidence. For future research, it is suggested to deepen these results on qualitative level.
\end{abstract}

\section{(c) (i) $(-)$}

Referencia de este artículo (APA):

Cabrera-García, V., Casas, K., Pardo, S. \& Rodríguez, D. (2017). Análisis de la resiliencia en personas divorciadas, según su nivel educativo e ingresos económicos. Psicogente, 20(37), 160-171. http://doi.org/10.17081/psico.20.37.2425

\footnotetext{
Artículo que se deriva del proyecto de investigación denominado Impacto psicosocial y económico del divorcio en Colombia, dirigido por Victoria Eugenia Cabrera-García, Investigadora principal del proyecto. Universidad de la Sabana, Bogotá D.C. Realizado desde enero 2014 hasta junio 2015.

1. Psicóloga, Profesora y Coordinadora de Investigación del Instituto de la Familia, Universidad de la Sabana.

Email: victoria.cabrera@unisabana.edu.co http://orcid.org/0000-0001-8907-5655

2. Magister en Formación de Asesoría Familiar y Gestión de programas para la Familia, Psicóloga, Universidad de la Sabana. Email: karlacaro@unisabana.edu.co http://orcid.org/0000-0002-6331-0044

3. Magíster en Formación de Asesoría Familiar y Gestión de programas para la Familia, Orientadora Escolar, Universidad de la Sabana. Email: sandraparher@unisabana.edu.co http://orcid.org/0000-0002-5325-1837

4. Magister en Formación de Asesoría Familiar y Gestión de programas para la Familia de la Universidad de la Sabana, Orientación Familiar. F.U.M Email:dianarodma@unisabana.edu.co http://orcid.org/0000-0002-7053-9146
} 


\section{Introducción}

A lo largo de la historia de la humanidad, el fenómeno de los conflictos humanos ha sido abordado desde distintas disciplinas. Hoy en día, los problemas continúan siendo consustanciales a la vida cotidiana, pero más trascendente aún es la forma en que se afrontan. Una de estas experiencias es el divorcio, el cual causa un profundo impacto en la sociedad, la familia y sus miembros. Aunque el divorcio cristaliza una situación que ya era conflictiva, incita a oficializar la ruptura de la pareja y el núcleo familiar (Pérez, Castillo \& Davins, 2006).

Cuando los conflictos entre los cónyuges se hacen cada vez más frecuentes y difíciles de solucionar, se considera el divorcio como una alternativa para resolver los problemas matrimoniales, según Jiménez, Amaris y Valle (2012). A partir de esta decisión entran en juego factores claves en la resolución y el restablecimiento de la vida de las personas.

Aunque los seres humanos cuentan con una capacidad inherente para sobreponerse ante situaciones desfavorables, para Gómez (2010), las situaciones adversas se afrontan mejor con adecuados niveles de resiliencia. Según Rutter (1993), la resiliencia es un término adaptado a las Ciencias Sociales para caracterizar aquellas personas que, a pesar de nacer y vivir en situaciones de alto riesgo, se desarrollan psicológicamente sanos y exitosos. Otros autores la definen como la capacidad humana universal para hacer frente a las adversidades de la vida, superarlas o, incluso, ser transformado positivamente por ellas (Martínez, 2011; Grotberg, 2003). La resiliencia es parte del proceso evolutivo y debe ser promovida desde la niñez (Grotberg, 1995). Para Kotliarenco, Mardones, Melillo y Suárez (2000) es el constructo que se implementa para representar las adaptaciones positivas en medio de la adversidad.
Por su parte, Vanistendael (1994) define la resiliencia como la capacidad de una persona o sistema social de enfrentar adecuadamente las dificultades, de una forma socialmente aceptable. Asimismo, Rutter (1993) explica que la resiliencia se ha caracterizado por un conjunto de procesos sociales e intrapsíquicos que posibilitan tener una vida sana, viviendo en un medio insano. Se han desarrollado estudios sobre resiliencia en niños y adolescentes en relación con diferentes tipos de adversidades como, por ejemplo, el maltrato (Henry, 1999), el abuso sexual (De Arce \& Aguayo, 2006) o la influencia de las guerras (Berk, 2002). Ahora bien, la resiliencia no solo ha centrado sus estudios en la infancia y la adolescencia, sino que también ha mostrado su validez en otros grupos de edad (Saavedra, 2005) y para otro tipo de adversidades aplicadas a la etapa adulta: atentados (Calderón, Espinosa \& Techio, 2004), desigualdad o violencia de género (Santana, 2010) y afrontamiento de enfermedades graves.

Estudios sobre la resiliencia muestran un énfasis investigativo tendiente a analizar el resultado del proceso de afrontamiento de una situación estresante, que permite al individuo mantener su equilibrio y mostrar una buena adaptación a pesar de estar expuesto a una dosis de adversidad significativa. Esta resistencia frente a la adversidad y la capacidad para construir conductas vitales positivas, con el fin de alcanzar niveles de calidad de vida adecuados, se conoce con el nombre de resiliencia (Kotliarenco, Cáceres \& Fontecilla, 1997). En este orden de ideas, y de acuerdo con los objetivos de esta investigación, la resiliencia podría ayudar a las personas a enfrentar situaciones estresantes, en este caso el evento del divorcio.

Según diferentes teóricos, el divorcio es un fenómeno indeseable para las personas que lo viven y para 
la sociedad entera, y no contribuye a la sostenibilidad social y económica de la familia (Cabrera, Docal, Muñoz \& Olaya, 2015; Amato, 2000).

En Colombia se ha incrementado el divorcio en los últimos años. Durante el tercer trimestre del 2014, se reportaron 17.644 registros de matrimonio frente a 4.881 divorcios en el mismo trimestre (Boletín estadístico notarial $\mathrm{N}^{\circ} 4$ de diciembre de 2014). Su vivencia afecta significativamente a los integrantes de las parejas y familias que lo experimentan. Es importante considerar que la severidad y duración de los efectos del divorcio varían de persona a persona, dependiendo de la presencia de una variedad de factores de protección (Amato, 2000). Entre estos factores se pueden considerar las características de resiliencia que tengan las personas. Algunos estudios han encontrado que la tristeza, angustia, depresión, consumo de alcohol y problemas de salud subsisten alrededor de dos o tres años después de la separación, lo cual se enmarca en el modelo de la crisis temporal.

Si bien es cierto que en otros países las personas divorciadas reciben apoyos de diferente índole, en el contexto nacional la situación no es igual, pues todavía se estigmatiza de manera negativa y con actitud de exclusión a las personas que están viviendo este fenómeno. En este orden de ideas, sería interesante investigar los niveles de resiliencia de las personas durante la ocurrencia del fenómeno del divorcio, teniendo en cuenta su nivel educativo e ingresos económicos para observar si estos recursos les ayudan a enfrentar mejor este evento.

\section{Concepción de la resiliencia}

Para efectos de este estudio se siguió la propuesta de Palomar y Gómez (2010), quienes contemplan cinco factores para evaluar la resiliencia. Estos son:
La fortaleza y confianza en sí mismo, que se define como el "sentimiento de adecuación, eficiencia y competencia en el afrontamiento de los retos y amenazas que, inevitablemente, se presentan en la vida de cualquier persona" (Palomar \& Gómez, 2010, p.11). Los ítems o reactivos que forman parte de este factor se refieren a la claridad que las personas tienen sobre sus objetivos y al esfuerzo que hacen por alcanzar sus metas. También se integra con la confianza que tiene la persona de alcanzar el éxito, al optimismo, la fortaleza y la tenacidad con la cual enfrentan los desafíos; mostrando recursos para hacer frente a los eventos estresantes o perturbadores. Es decir, que la fortaleza le permite al individuo adaptarse y sobreponerse a condiciones adversas. Por su parte, Seligman (2003) agrega que las fortalezas actúan a modo de barrera contra la desgracia y los trastornos psicológicos, y pueden ser la clave para aumentar la capacidad de recuperación en tiempo de crisis. De esta manera, al pasar por la experiencia del divorcio se pueden experimentar sentimientos de frustración personal y tristeza debidos a la dificultad de continuar con las metas, generando incertidumbre de alcanzar los objetivos del día a día.

La competencia social se refiere a la "capacidad o habilidad del sujeto para interactuar exitosamente con su ambiente y lograr la satisfacción legítima de sus necesidades, al ejercer determinada influencia sobre sus semejantes. Es una cualidad muy relacionada con la salud, el ajuste emocional y el bienestar de los individuos" (Palomar \& Gómez, 2010, p.11). Las competencias sociales son aquellas aptitudes necesarias para tener un comportamiento adecuado y positivo que permita afrontar eficazmente los retos de la vida diaria. Son comportamientos o tipos de pensamientos que llevan a resolver una situación de manera efectiva, es decir, aceptable para el propio sujeto y para el contexto social en el que está. 
Un individuo con déficit en sus habilidades sociales básicas tiene mayores probabilidades de afrontar problemas emocionales y dificultades en su competencia social, lo que lo hace más vulnerable a causa de la frustración de necesidades como seguridad, aceptación, realización; y puede provocar que obtenga menos reforzamiento social en general, lo que, además, daña su autoestima y su sentimiento de identidad. Es decir, la falta de competencia social en medio de una situación tan estresante como el divorcio puede provocar baja autoestima y, por tanto, altos niveles de malestar.

El apoyo familiar hace referencia a las relaciones familiares y al apoyo que brinda la familia a quien atraviesa una situación de impacto o crisis. En este sentido, Palomar y Gómez (2010) se refieren "al tiempo que comparten los miembros de la familia, la lealtad que existe entre ellos y las fortalezas de los lazos familiares" (p.11). Por su parte, Seccombe (2000) resalta que los factores familiares y sociales son un soporte y, a su vez, un mitigador de los efectos negativos del estrés, promueven las habilidades y la autoestima necesaria para sobreponerse a la adversidad. Las relaciones positivas y mayormente las del contexto familiar se identifican como promotoras de autoestima y autosuficiencia necesarias en momentos de crisis personal. En este caso, el apoyo familiar, en medio del divorcio y la tristeza, permite lidiar con los sentimientos negativos.

Al respecto, Athié y Gallegos (2009), en su investigación sobre la relación entre la resiliencia y el funcionamiento familiar, encontraron una relación entre el factor de resiliencia que orienta a las personas hacia el futuro y los factores del funcionamiento familiar, es decir, las personas son más resilientes cuando cuentan con comunicación, cohesión familiar, tiempo compartido, roles compartidos, autoridad, fomento de la auto- nomía e independencia. Continuando con lo expuesto por Athié y Gallegos (2009), y específicamente hablando de aquellos factores protectores que se dan producto de la relación de apoyo entre padres e hijos, se muestra, en primer lugar, que el sistema de creencias familiares, que da un significado a situaciones de crisis como la separación o el divorcio, puede o no facilitar la superación de este evento. En segundo lugar, se hace referencia a la estructura familiar y la forma de organización y liderazgo que en ella se observa, así el soporte mutuo será de mayor ayuda. Se destaca a la familia como sistema de influencia a nivel preventivo, lo cual permite desarrollar en sus integrantes factores de protección al proporcionar herramientas con las cuales enfrentar adversidades, desde el establecimiento de relaciones de confianza y de cuidado, promoviendo la cohesión y el apoyo. El ambiente familiar es uno de los factores más importantes para que las personas desarrollen un mejor nivel de resiliencia.

En cuanto al apoyo social, este se refiere a "los vínculos existentes entre el sujeto y un conjunto de personas con las cuales es factible el intercambio de comunicación, solidaridad y confianza" (Palomar \& Gómez, 2010, p.11). Briceño (2014) cita a Melillo y Suárez (2004), quienes definen el apoyo social por la presencia o ausencia relativa de recursos de apoyo psicológico provenientes de otras personas significativas. Conjunto de recursos humanos y materiales con que cuenta un individuo o familia para superar una determinada crisis, enfermedad, condiciones económicas difíciles, rupturas familiares, divorcio. Este factor se centra, principalmente, en los amigos y hace referencia al hecho de contar en los momentos difíciles con personas que puedan ayudar, que se puedan preocupar y den aliento (Palomar \& Gómez, 2010). Las personas que no tengan amigos de referencia o de apoyo pueden presentar episodios de 
profunda soledad y tristeza en una situación de crisis como el divorcio.

Finalmente, en cuanto a la estructura, esta se refiere a la facilidad que muestran las personas en la organización y el orden de su vida a partir de las reglas y las actividades que llevan a cabo (Palomar \& Gómez, 2010). Los individuos resilientes son sistemáticos y organizados. Aun cuando al afrontar sus problemas sean flexibles, contar con un proyecto de vida claro y tener facilidad para desarrollarlo es un aspecto importante en el momento en el que la persona deba continuar su vida después de la situación que lo haya impactado. Es importante aclarar que la resiliencia no protege a las personas de eventos negativos o impactantes, sino que las ayuda en el proceso de reestructuración de sus vidas (Friborg, Hjemdal, Rosenvinge \& Martinussen, 2001).

Según lo anterior, la persona que logra planificar, que muestra disponibilidad y autocontrol con relación a reglas, rutinas y que prefiere la planeación de sus actividades al considerar que es mejor la calidad de sus trabajos con metas claras y buena organización; tendría mayor capacidad para afrontar las situaciones que impacten o generen crisis en su vida. La planificación y la organización se asocian con la autorrealización y el crecimiento, de igual manera, ese crecimiento tiene alta incidencia en la capacidad de afrontamiento de una persona ante su separación o divorcio (Aciego, Domínguez \& Hernández, 2005). Asimismo, el divorcio puede generar un deterioro en el normal funcionamiento de la dinámica personal y laboral e interferir con las rutinas y hábitos sociales.

Algunos factores sociodemográficos como el nivel educativo y el nivel de ingresos pueden mostrar diferencias en el nivel de resiliencia.
Según Delgado (2014), para la Fundación para la Educación Superior y el Desarrollo (FEDESARROLLO), el nivel educativo se ha dividido en cuatro niveles: la educación preescolar, la educación básica, la educación media y la educación superior. Martínez (2011) en su estudio "La educación para una sociedad resiliente", indica que la resiliencia constituye una competencia personal imprescindible para afrontar con éxito las situaciones y experiencias dolorosas y problemáticas, proponiendo que el sistema educativo y las familias deben ser responsables de la educación de la competencia en resiliencia. La resiliencia así concebida se convierte en una meta-competencia crítica y transversal en los tiempos actuales, que debe abordarse en todos los niveles de la educación, desde la educación infantil hasta la universidad.

Por otro lado, Cabrera, González, Vargas y Franco (2012), en su investigación, encontraron que los problemas académicos como el bajo rendimiento, el fracaso y la deserción escolar tienen una relación directa con la conducta transgresora o antisocial en menores de edad con dificultades en el desarrollo de habilidades cognitivas, lo cual contribuye, como factor de vulnerabilidad, a la conducta delictiva en la adolescencia y, posteriormente, a lo largo del ciclo vital. De otra manera, un mayor nivel educativo puede promover habilidades cognoscitivas tendientes a una mejor adaptación al medio y, por tanto, la capacidad de enfrentar las situaciones adversas que este provee.

En su estudio, Baum (2003) expone que existe una serie de elementos que mediatizan la adaptación de las parejas ante un divorcio, dentro de ellos se destaca el nivel socioeconómico y educativo de los cónyuges, encontrando al respecto que a mayor estatus socioeconómico y nivel educativo de los involucrados, mejor fue la 
forma en que ambos cumplían sus funciones parentales y cooperaban entre ellos.

Por su parte, los ingresos económicos hacen referencia a todas las entradas monetarias que percibe una persona, una familia, una empresa, una organización, un gobierno. De acuerdo con Amato (2000) en cuanto a los efectos del divorcio y los factores de riesgo y a las diferencias por género, las mujeres presentan mayores consecuencias económicas, ya que perciben que su nivel de vida disminuye, lo cual parece estar relacionado con historias laborales generalmente más discontinuas, un mayor conflicto trabajo-familia y la discriminación laboral y salarial. Por su parte, Morgado, Gonzales y Jiménez (2003), citados por López (2009), encontraron que el $70 \%$ de las familias monoparentales (generalmente constituidas tras el divorcio y encabezadas por una mujer) no contaban con ingresos suficientes.

La mayor probabilidad de que cualquiera de los cónyuges pida el divorcio ocurre cuando sus ingresos económicos son casi iguales y son pocas las obligaciones financieras recíprocas (Rogers, 2004).

Continuando con lo expuesto, un estudio que mide la incidencia de factores como sexo, edad, ingreso, estado civil y norma de grupo respecto a la actitud hacia el aborto inducido obtuvo entre sus resultados emociones negativas hacia la probabilidad de llevar a cabo la solicitud de interrupción del embarazo. Además, la desaprobación parece incrementarse en mujeres, adultas jóvenes, con ingresos económicos que superan el mínimo y en proceso de separación o divorcio, así como en aquellas pertenecientes a grupos liberales o progresistas, según García, Carreón, Hernández, Bautista y Méndez (2012). Es decir, con mayores ingresos económicos al mí- nimo legal vigente existe una menor probabilidad de interrumpir el proceso de embarazo y afrontar de manera adecuada la nueva experiencia.

En este orden de ideas, la pregunta que guía este estudio es: ¿existen diferencias significativas en los factores de resiliencia en medio del divorcio respecto al nivel educativo e ingresos económicos?

\section{Método}

\section{Tipo de investigación}

Estudio cuantitativo transversal, descriptivo. Con el fin de responder a las preguntas de investigación se corrieron Análisis de Varianza (ANOVA) y la interacción de las variables demográficas, nivel educativo e ingresos económicos.

\section{Participantes}

Se contó con 139 personas de nacionalidad colombiana que se habían divorciado en un periodo no mayor a tres años (según Sabatelli \& Ripoll (2004), el duelo del divorcio dura aproximadamente tres años) y que tuvieran al menos un hijo, 77 (55,4 \%) de sexo femenino y $62(44,6 \%)$ de sexo masculino. Para el nivel educativo, los participantes se organizaron en dos grupos así: grupo 1: estudios de primaria, secundaria y media con 48,2 \%; grupo 2: algunos estudios universitarios, pregrado y posgrado con 51,8 \%. Para los ingresos económicos, el primer grupo se conformó con las personas que tienen ingresos económicos hasta $\$ 1.800 .000$ y que corresponde al 60,4\%; el segundo grupo corresponde a personas con ingresos económicos superiores a $\$ 1.800 .000$, que equivale al 39,6\% de los participantes. 


\section{Instrumentos}

Se diseñó un cuestionario que recogía información sobre el sexo del participante, el nivel educativo, los ingresos económicos, entre otras variables demográficas, así como se aplicó la Escala de Resiliencia Mexicana RESI-M, desarrollada por Palomar y Gómez (2010). Esta escala se compone de 43 ítems divididos en cinco factores: Fortaleza y confianza en sí mismo, $(\alpha=.92)$; competencia social, $(\alpha=.87)$; apoyo familiar, $(\alpha=.87)$; apoyo social, $(\alpha=.84)$; y estructura, $(\alpha=.79)$. Para efectos de este estudio, en el factor competencia social el alpha fue de .89; apoyo familiar, alpha de .90; apoyo social, alpha de .88 y en el factor estructura, alpha de .73 . En el factor fortaleza y confianza en sí mismo se obtuvo el mismo nivel de confiabilidad que en la escala original. La resiliencia total es el promedio de todos los factores nombrados anteriormente.

\section{Procedimiento}

Para la recolección de la información se diseñó una aplicación para Internet desde Google Application con el objetivo de recibir los cuestionarios en línea, logrando así reducir el margen de error y garantizando mayor confidencialidad de los datos. Los participantes se contactaron a través de la estrategia "bola de nieve" que busca contactar un participante a través de otro. Se explicaron los objetivos del estudio y que la información sería utilizada de manera confidencial y anónima, solo para los fines de la investigación. La participación fue voluntaria con previa firma del consentimiento informado.

\section{Resultados}

Los análisis de datos se corrieron a través del programa SPSS versión 21.
En primer lugar, se analizaron los estadísticos descriptivos de las variables de resiliencia del estudio.

Tabla 1. Estadísticos descriptivos de las variables de estudio

\begin{tabular}{cccccc}
\hline $\begin{array}{c}\text { Fortaleza y } \\
\text { confianza } \\
\text { en sí mismo }\end{array}$ & $\begin{array}{c}\text { Compe- } \\
\text { tencia } \\
\text { social }\end{array}$ & $\begin{array}{c}\text { Apoyo } \\
\text { familiar }\end{array}$ & $\begin{array}{c}\text { Apoyo } \\
\text { social }\end{array}$ & Estructura & $\begin{array}{c}\text { Resiliencia } \\
\text { total }\end{array}$ \\
\hline $3,39(.44)$ & $3,23(.56)$ & $3,41(.58)$ & $3,54(.47)$ & $3,15(.52)$ & $3,34(.37)$ \\
\hline Nota: Media (Desviación estándar) & & &
\end{tabular}

La Tabla 1 muestra que los participantes del estudio obtuvieron el puntaje más alto en apoyo social, seguido por apoyo familiar. En contraste, la media más baja se obtuvo en estructura, es decir, en la organización y el orden de la vida de las personas a partir de las reglas y las actividades que llevan a cabo.

\section{Análisis de diferencias de medias en la resiliencia de acuerdo con el nivel educativo}

Se encontraron diferencias significativas según el nivel educativo en competencia social $(F(1,138)=4.51$; $p=.03)$, es decir, las personas con estudios universitarios y de posgrado $(M=3,33)$ muestran mayores habilidades en equilibrarse con los otros y con su ambiente que las que tienen estudios básicos $(M=3,13)$. Asimismo, se encontraron diferencias en apoyo social $(\mathrm{F}(1,138)=6.55$; $p=.01)$, es decir, las personas de educación universitaria y posgrado $(M=3,64)$ presentan mayor nivel de apoyo de los amigos que los de educación básica $(M=3,43)$.

No se encontraron diferencias en la fortaleza y confianza en sí mismo $(\mathrm{F}(1,138)=1.69$; $p=.19)$, tampoco hubo diferencias significativas en apoyo familiar $(\mathrm{F}(1,138)=.004 ; p=.95)$, en estructura $(\mathrm{F}(1,138)=.34$; $p=.56)$, ni en la resiliencia total $(\mathrm{F}(1,138)=3.08$; $p=.08)$. Esto quiere decir que las personas con nivel educativo básico y nivel educativo universitario pueden contar con fortaleza y confianza en sí mismas, con apoyo familiar, 
capacidad de organizarse en sus tareas y rutinas, como en su resiliencia total.

\section{Análisis de diferencias de medias en la resiliencia de acuerdo con los ingresos económicos}

Se encontraron diferencias significativas en fortaleza y confianza en sí mismo $(\mathrm{F}(1,138)=12,78 ; p=.000)$, es decir, que las personas que tienen ingresos económicos superiores a $\$ 1.800 .000$ evidencian mayores niveles de aceptación de sí mismo y consecución de logros $(M=3,55)$, en contraste con los que cuentan con ingresos económicos inferiores a $\$ 1.800 .000(\mathrm{M}=3,29)$. En competencia social $(F(1,138)=9,79$; $p=.002)$, los resultados demuestran que las personas agrupadas en los ingresos económicos superiores a $\$ 1.800 .000$ presentan mayores competencias para equilibrarse a las personas de su entorno $(M=3,41)$, en contraste con los que ganan hasta $\$ 1.800 .000(\mathrm{M}=3,11)$. En cuanto el apoyo familiar $(\mathrm{F}(1,138)=6,92 ; p=.009)$, también se encontraron diferencias significativas para las personas que tienen ingresos económicos mayores a $\$ 1.800 .000(\mathrm{M}=3,57)$, es decir, que existe mayor lealtad entre la familia en contraste con las personas que tienen ingresos económicos menores a $\$ 1.800 .000(\mathrm{M}=3,31)$. En cuanto a estructura también se obtuvieron resultados significativos $(F(1,138)$ $=9,76 ; p=.002)$ al observarse que las personas que tienen ingresos económicos por encima de $\$ 1.800 .000$ dan continuidad a sus proyectos de vida $(M=3,32)$, en contraste con las personas que tienen ingresos económicos por debajo de $\$ 1.800 .000(\mathrm{M}=3,04)$. Respecto al nivel de resiliencia total también se encontraron diferencias significativas $(\mathrm{F}(1,138)=15,77 ; p=.000)$, es decir, entre las personas con ingresos económicos superiores a $\$ 1.800 .000(\mathrm{M}=3,49)$ y las personas con ingresos económicos menores a $\$ 1.800 .000(\mathrm{M}=3,25)$.

No se encontraron diferencias significativas en apoyo social $(\mathrm{F}(1,138)=2.95 ; p=.088)$, es decir, que las personas con ingresos económicos inferiores y superiores a $\$ 1.800 .000$ obtuvieron apoyo de sus amigos en su proceso de divorcio.

\section{Efecto de interacción entre nivel educativo e ingresos económicos en las distintas dimensiones de la resiliencia}

En cuanto a la fortaleza y confianza en sí mismo se encontró un efecto de interacción entre el nivel educativo y el nivel de ingresos $(\mathrm{F}(3,138)=5.39 ; p=.02), \alpha 2$ $=.038$, es decir, que las personas con un nivel educativo básico y que ganan más de $\$ 1.800 .000$ (M=3.67) demuestran mayores niveles en esta dimensión, seguidos por los que tienen una educación universitaria y ganan más de $\$ 1.800 .000(\mathrm{M}=3.49)$. Le siguen los que tienen educación universitaria y ganan menos de $\$ 1.800 .000$, $(\mathrm{M}=3.38)$ y, finalmente, los que tienen educación básica y ganan menos de $\$ 1.800 .000$ son los que demuestran menor fortaleza y seguridad en sí mismos $(M=3,22)$ (ver Figura 1).

No se encontró un efecto de interacción entre el nivel educativo e ingresos económicos en el resto de factores de la resiliencia.

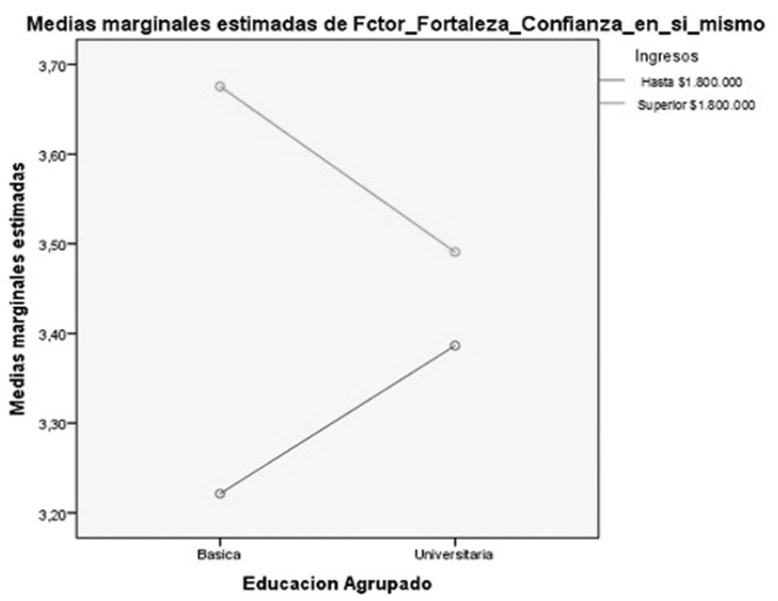

Figura 1. Efecto de interacción entre el nivel educativo e ingresos económicos en la Fortaleza y confianza en sí mismo 


\section{Discusión}

El objetivo del estudio fue identificar los niveles de resiliencia en personas divorciadas de acuerdo con el nivel educativo y los ingresos económicos. Los resultados evidenciaron que sí existen diferencias significativas según las variables sociodemográficas estudiadas en cuanto al nivel de resiliencia en medio del divorcio.

Las personas con estudios universitarios de pregrado y/o posgrado presentaron mayores niveles de resiliencia en competencia social y apoyo social que las que tienen educación básica, es decir, que poseen más capacidades para interactuar exitosamente con otras personas y con su ambiente, así como mayor apoyo de sus amigos, recursos que proporcionan comunicación, solidaridad y confianza en medio del divorcio. No se trata solo de contar con personas, sino que estas se caracterizan porque pueden ayudar y dar aliento. Este resultado concuerda con lo encontrado por Cabrera et al. (2012), quienes consideran que las personas con mayor nivel educativo tienen mejor adaptación ante las adversidades. Siguiendo las ideas de Martínez (2011) y Grotberg (2003), y en línea con los resultados de esta investigación, las personas divorciadas pueden desarrollar competencias sociales y contar con apoyo social para hacer frente a su proceso de separación e, incluso, ser transformadas positivamente por este. Aunque los resultados están dirigidos a las personas de alto nivel educativo, también se podría trabajar, desde edades tempranas, con personas de bajo nivel educativo con el objetivo de fomentar procesos de resiliencia en ellas.

Los aportes de Kotliarenco et al. (1997), que hacen énfasis en los estudios de Bradley, Whiteside, Mundfrom, Casey, Kelleher y Pope (1994) sobre los riesgos de los niños y niñas con dificultad económica, quienes son más propensos a contraer enfermedades físicas con más frecuencia y presentar deficiencia en el apoyo social; coinciden con los resultados del presente estudio. Es decir, los resultados permiten afirmar que los niveles de resiliencia son altos en las personas que tienen mayores ingresos económicos, como se evidencia en este estudio, en la fortaleza y confianza en sí mismo, en la competencia social, el apoyo familiar, la estructura y la resiliencia total. Esto quiere decir que son personas que a pesar de afrontar la crisis del divorcio tienen claridad sobre sus objetivos, presentan un equilibrio social con buen ajuste emocional, existe lealtad entre la familia, hay continuidad en los hábitos y rutinas sociales, en contraste con las personas que tienen menos ingresos económicos. Esto lleva a pensar que un buen nivel de ingresos económicos puede ayudar a enfrentar una situación adversa como el divorcio. Algunos investigadores explican que, en medio del divorcio, los esposos que poseen pocos recursos económicos experimentan una disminución en su felicidad. A su vez, los asuntos económicos tienden a ser una causa importante de ruptura matrimonial (Rogers, 2004).

Por otro lado, fue interesante analizar que los ingresos económicos y el nivel educativo de las personas jugaron, simultáneamente, un papel significativo en el nivel de resiliencia en medio del divorcio. Es decir, que el efecto de los ingresos económicos en la fortaleza y confianza en sí mismo, indicadores de resiliencia, depende de los niveles de formación educativa de las personas, quienes se sienten competentes y eficientes al afrontar el divorcio de acuerdo con su nivel de ingresos y nivel educativo, simultáneamente.

Los resultados de este estudio abren un debate interesante en la psicología y en el análisis de las situaciones adversas. La cuestión por analizar consiste en si las personas pueden hacer frente al divorcio y ajustarse a este gracias a un alto nivel de ingresos económicos, a contar con características de resiliencia o gracias a un 
alto nivel educativo. Como lo explica Grotberg (2003), es necesario contar con factores protectores en medio de la vivencia de una adversidad, en este caso el divorcio. Sería interesante continuar este debate en futuras investigaciones con el fin de analizar el peso o la importancia de estos factores sociodemográficos en el afrontamiento de este evento adverso, y considerar el papel que juegan otros factores como las características de personalidad del divorciado, el sexo o el cargo laboral en el que trabaje.

Estos resultados pretendieron contribuir al conocimiento y estudio del fenómeno del divorcio desde la Psicología, y así iluminar otras disciplinas en el estudio de este evento. Asimismo, aportar al ejercicio de los profesionales que trabajan con parejas que están viviendo procesos de divorcio, con el fin de fortalecer sus niveles de resiliencia de manera intrapersonal en cada una de las dimensiones contempladas por Palomar y Gómez (2010).

Se sugiere ampliar en próximas investigaciones aspectos como las estrategias de afrontamiento de cara al divorcio, realizar estudios cualitativos para profundizar y comprender este evento en la vida de las personas, así como elaborar programas preventivos y de intervención para promover la resiliencia de las personas desde los niveles básicos de educación, que permitan desarrollar habilidades que contribuyan a enfrentar diferentes situaciones adversas que puedan ocurrir en la vida.

De esta misma forma, hay un marcado interés porque los resultados de esta investigación proporcionen herramientas útiles a las parejas, frente a la comprensión de la importancia de acceder tanto a la educación básica como a la educación superior que facilite una mayor formación y la consecución de mayores ingresos económi- cos para alcanzar niveles más altos de bienestar y calidad de vida.

Finalmente, se sugiere que desde las diferentes disciplinas científicas se siga fortaleciendo y profundizando en el estudio de la resiliencia desde una metodología cuantitativa y cualitativa, con el fin de enfrentar las diferentes adversidades que se puedan presentar en el matrimonio y en la familia.

\section{Referencias}

Aciego de Mendoza, R., Domínguez, R. \& Hernández, P. (2005). Consistencia interna y estructura factorial de un cuestionario sobre autorrealización y crecimiento personal. Recuperado de: http:// www.psicothema.com/pdf/3077.pdf

Amato, P. (2000). The consequences of divorce for adults and children. Journal of Marriage and Family, 62, 1269-1287. Recuperado de: http:// onlinelibrary.wiley.com/doi/10.1111/j.17413737.2000.01269.x/abstract.

Athié, D. \& Gallegos, P. (2009). Relación entre la resiliencia y el funcionamiento familiar. Psicología Iberoamericana, 17(1), 5-14. Recuperado de: http:// www.redalyc.org/articulo.oa?id=133912613002

Baum, N. (2003). Divorce process variables and the coparental relationship and parental role fulfillment of divorce parents. Family Process, 42(1), 117-131.

Berk, J. (2002). Trauma y resiliencia durante la guerra: una mirada a los niños y a los trabajadores de ayuda humanitaria en Bosnia. Psicoanálisis: Revista de la Asociación Psicoanalitica de Buenos Aires, 24(1/2), 45-65. 
Briceño, M. (2014). Resiliencia en la gestión de las organizaciones. Revista de Investigación en Administración e Ingeniería, 2(54). Recuperado de: http:// service.udes.edu.co/revistas/index.php/aibi/article/view/0201/020105.pdf

Cabrera, G., González, J., Vargas, L. \& Franco, L. (2012). Conducta antisocial y delictiva en adolescentes de un centro de reclusión en el Quindío, periodo 2008-2010. Revista Psicogente, 15. Recuperado de: http://publicaciones.unisimonbolivar.edu. co:82/rdigital/psicogente/index.php/psicogente/article/viewFile/315/307

Cabrera, V., Docal, M., Muñoz, D. \& Olaya, J. (2015). El divorcio ifracaso u oportunidad? Bogotá: Universidad de la Sabana.

Calderón, A., Espinosa A. \& Techio, E. (2004). Resiliencia, afrontamiento, bienestar psicológico y clima socio-emocional después de los atentados del 11 de marzo. Ansiedad y Estrés, 10(2/3), 265-276.

De Arce, J. \& Aguayo, P. (2006). Abuso sexual intrafamiliar: prevalencia y características en jóvenes de $3^{\circ}$ medio de liceos municipalizados de Chillán, Chile. Theoria: Ciencia, Arte y Humanidades, 15(1), 79-85.

Delgado, M. (2014). La educación básica y media en Colombia: Retos en equidad y calidad. Informe final de la Fundación para la Educación Superior y el Desarrollo (FEDESARROLLO). Recuperado de: http://www.repository.fedesarrollo.org.co/ bitstream/11445/190/1/La-educaci\%C3\%B3nb\%C3\%A1sica-y-media-en-Colombia-retos-enequidad-y-calidad-KAS.pdf
Friborg, O., Hjemdal, O., Rosenvinge, J. \& Martinussen, M. (2001). A new rating scale for adult resilience: What are the central protective resources behind healthy adjustment? International Journal of Methods in Psychiatric Research, 12(2), 65-76.

García, C., Carreón, J., Hernández, J., Bautista, M. \& Méndez, A. (2012). Determinantes socioeconómicos y demográficos de las actitudes hacia el aborto. Revista Psicogente, 15. Recuperado de: http:// publicaciones.unisimonbolivar.edu.co:82/rdigi$\mathrm{tal} / \mathrm{psicogente} /$ index.php/psicogente/article/ view/328/320

Gómez, B. (2010). Resiliencia individual y familiar. Recuperado de: http://www.avntfvntf.com/imagenes/biblioteca/G\%C3\%B3mez,\%20B.\%20 Trab.\%203\%C2\% A\%20BI\%2009-10.pdf

Grotberg, E. (1995). The Internacional Resilience Project: Promoting Resilience in Children. Wisconsin: Universidad de Wisconsin.

Grotberg, E. (2003). La resiliencia en el mundo de hoy. Barcelona, España: Editorial Gedisa S.A.

Henry, D. (1999). Resilience in Maltreated Children: Implications for Special Needs Adoption. Child Welfare, 78(5), 519-540.

Jiménez, M., Amaris, M. \& Valle, M. (2012). Afrontamiento en crisis familiares: el caso del divorcio cuando se tienen hijos adolescentes. Salud Uninorte Barranquilla, 28(1), 99-112. Recuperado el 5 de agosto de 2014 de http://rcientificas.uninorte.edu.co/index.php/salud/article/down$\operatorname{load} / 3900 / 2857$ 
Kotliarenco, M., Cáceres, I. \& Fontecilla, M. (1997). Estado de Arte en Resiliencia. Organización Panamericana de la Salud. CEANIM. Santiago de Chile: OPS.

Kotliarenco, M., Mardones, F., Melillo, A. \& Suárez, N. (2000). Actualizaciones en resiliencia. Buenos Aires: Universidad Nacional de Lanús, Fundación Bernard van Leer.

López, L. (2009). El sistema familiar ante el divorcio: factores de riesgo y protección y programas de intervención. Universidad de La Coruña, España. Recuperado de: http://www.researchgate.net/publication/233598082_El_sistema_familiar_ante_el_ divorcio_factores_de_riesgo_y_proteccin_y_programas_de_intervencin_The_family_system_ upon_divorce_Risk_and_protective_factors_ and_intervention_programmes

Martínez, J. (2011). La educación para una sociedad resiliente. Contribuciones a las Ciencias Sociales, Universidad de la Laguna, España. Recuperado de: http://www.eumed.net/rev/cccss/14/jamg2. html

Palomar, J. \& Gómez, N. (2010). Desarrollo de una escala de medición de la resiliencia con mexicanos (RESI-M) interdisciplinaria. Red de Revistas Cientificas de América Latina, el Caribe, España y Portugal, 27(1), 7-22. Recuperado el 17 de junio de 2014 de http://www.redalyc.org: http://www.redalyc.org/ articulo.oa? $\mathrm{id}=18014748002$

Pérez, T., Castillo, J. \& Davins, M. (2006). El divorcio. Compendio parejas en conflicto. Barcelona: Paidós.
Rutter, M. (1993). Resilience: Some conceptual considerations. Journal of Adolescent Health, 14, 626-631.

Saavedra, E. (2005). Resiliencia: La historia de Ana y Luis. Liberabit. Revista de Psicología, 11, 91-101.

Sabatelli, R.M. \& Ripoll, K. (2004). Variations in marriage over time: An ecological/Exchange perspective. En M. Coleman \& L.H. Ganong. (Eds.), Handbook of contemporary families: considering the past, contemplating the future (pp.79-95). Estados Unidos: Sage Publishing.

Santana, J. (2010). La formación para el trabajo social en contextos de desigualdad de género y violencia contra las mujeres. Portularia, 91-99.

Seccombe, K. (2000). Families in poverty in the 1990s: Trends, causes, consequences and lessons learned. Journal of Marriage and the Family, 62, 1094-1113. Recuperado de: http://www. readcube.com/articles/10.1111\% $2 \mathrm{Fj} .1741$ 3737.2000.01094.x?r3_referer=wol\& tracking_ a c t i o n $=$ previ e w _ click \& s how checkout $=1 \&$ purchase_referrer $=$ onlinelibrary. wiley.com\& purchase_site_license=LICENSE_ DENIED

Seligman, M. (2003). Positive pychology: fundamental assumptions. Psychologist, 16(3), 126-127.

Vanistendael, S. (1994). Resilience: a few key issues. Malta: International Catholic Child Bureau.

Rogers, C. (2004). Grupos de encuentro. Argentina: Amorrortu. 